MODELING, IDENTIFICATION AND CONTROL, 2003, vOL. 24, No. 2, 87-97

doi:10.4173/mic.2003.22

\title{
Fusion of Radar Tracks, Reports and Plans
}

\section{O. M. MEVASSVIK*† and A. LØKKA $\ddagger$}

Keywords: Maritime surveillance, multiple hypothesis testing, constraint programming

\begin{abstract}
This paper suggests a method that utilizes non-real time information as an aid to improve maritime surveillance. Under certain conditions vessels move in accordance with preplanned routes and possibly also report their own position at certain positions or at certain times during the voyage. The method proposed consists of a statistical route model that describes the movement of the vessel, and includes refinement of the estimated movement based upon reports on the vessel. The estimated movement is then associated with radar tracks using multiple hypothesis techniques. This is due to the fact that the number of vessels with known route plans is small compared to the total number of vessels in an area. The associated radar tracks are also used to improve the estimated movement of the vessels. The problem of generating possible solutions and selecting the best hypothesis is formulated as a constraint satisfaction problem and implemented using a constraint programming technique.
\end{abstract}

\section{Introduction}

The work reported in this paper has been conducted as a part of a project concerning naval command and control (C2) systems at the Norwegian Defence Research Establishment (FFI).

A major focus in the project is maritime surveillance, including picture production which comprises collection, processing and distribution of data. As a part of this there is an activity examining algorithms to support the picture production process. The situation pictures considered are used for force level planning and decision aids. This paper describes an algorithm that utilises a certain class of non-real time information, namely a priori information on the movement of a vessel, and optionally position reports on the vessel during the voyage.

The problem considered is similar to associating Flight Plans to tracks in Air Traffic Control.

\subsection{Maritime picture production}

Maritime surface surveillance includes searching large areas with several types of sensors and sensor platforms, including maritime patrol aircrafts, shorebased radars, naval ships, maritime helicopters and radar satellites. Due to the nature of the task, the classification of a track (deciding the type of a vessel) is hard. You also want to do classification at a distance beyond visible range due to the limited number of surveillance assets available.

Prepared for presentation at FUSION 2000, 3rd International Conference on Information Fusion, 10-13 July, Paris, France.

$\dagger$ Division for Electronics, Norwegian Defence Research Establishment, PO Box 25, NO-2027 Kjeller, Norway. E-mail: Ole-Martin.Mevassvik@ffi.no

$\Varangle$ Division for Electronics, Norwegian Defence Research Establishment, PO Box 25, NO-2027 Kjeller, Norway.

* Author to whom correspondence should be addressed. 
The conditions mentioned above motivate for using all potential useful information in the surveillance process. Even though the spatial uncertainty associated with the information in a non-real time report may be large, it may still be valuable for classification and identification. This is due to the fact that identity is at static property of an object, and old information may thus be valuable.

Current systems for maritime picture production are mainly based on real time sources and have limited support for the use of non-real time information. There are several reasons for this. One important reason is that it is hard to assess the quality of the data in the reports, and furthermore, the uncertainties may be large and thus generate an association problem with the rest of the situation picture.

There are several types of non-real time information applicable to maritime picture production. This paper considers the case where we have information on the planned movement of a vessel, in addition to position reports (with no association uncertainty) on the vessel during the journey. These reports are sporadic, separated by several hours, and time late. Some possible information sources are discussed in chapter 2 .

\section{Possible information sources}

There are several types of information available that may be used to support the picture production process, and thus enhance the quality of the maritime picture.

The information can be divided in the following categories:

- Information on the planned movement of a vessel which optionally reports its own position. The route plan includes which harbours to visit or a detailed sailing route including positions and a time plan.

- Estimated movements based on e.g. intelligence. These can be associated with incidents or detections reported by own forces.

- Reports from own units or civilian authorities, e.g. from Harbour Authorities on vessels that are calling at a harbour.

- Information about public transport services (ferries etc) that run at regular intervals and follow specific routes.

The usefulness of the various information sources is not only depending on the degree of uncertainty in the data, but also the availability of a proper quality assessment.

It is not obvious how to integrate the information considered into the situation picture. One option is to present the info separately used as an aid to enhance situation awareness. Available tools in present systems are typical furthest-on-circles and PIM-tracks (Position Intended Movement). Care should be taken if one choose to integrate the result into the situation picture and it is also important to communicate the uncertainty with the user. Note that we also have chosen to differ between cooperative information, originating from the vessel itself, and non-cooperative information (e.g. from intelligence).

\section{Brief overview of the method developed}

This chapter gives an introduction to, and the idea behind, the method that has been developed. The method is based upon these basic steps:

- Simulate the voyage of a vessel based on a known (or estimated) route plan. 
- Improve the position estimate given by the route plan with position reports on the vessel.

- Associate the unknown radar tracks to the simulated movement of a vessel using a multiple hypothesis approach.

- Use the position estimates given by radar tracks to improve the position estimate.

An important aspect is the management of uncertainty, which has been thoroughly considered in the development of the route model. The various bullets above are further described in the following chapters.

\section{Route model}

The route model is based upon estimated time of departure, speed and a fairway that covers the movement of the vessel. An estimate of the time of arrival would be equivalent to a speed estimate. Both uniform and normal distributions of the speed and time estimates have been considered.

The route model can be separated into two parts. The first part is a geometric description of the most likely route given by a certain coordinate system. The second part describes movement and uncertainty in this coordinate system. We decided to keep the model simple since the given information is rather imprecise. A more detailed model therefore yields little improvement, but requires more computation time.

\subsection{Coordinate system}

It is assumed that the actual movement with high probability will stay inside a fairway described by a sequence of line segments $\left\{L_{i}\right\}$ (see Figure 1). The 'expected' route or mean route is then the sequence of line segments given as the straight lines connecting the mean points of the line segments (shown as double lines in Figure 1) specifying the fairway.

Every point $\mathrm{p}$ in the fairway is then identified with some point $\operatorname{Proj}(p)$ on the expected route. The natural way to choose the point $\operatorname{Proj}(p)$ is to let $\operatorname{Proj}(p)$ be the point given by the euclidean projection of $p$ on the local line segment. This defines a one dimensional coordinate system with scale given as the distance along the line segments.

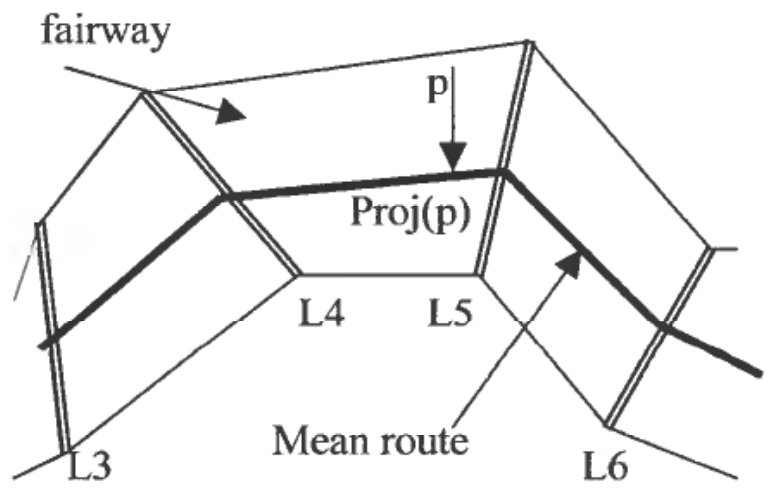

Figure 1. The route coordinate system. 
Geometric considerations of the fairway also provide information about probable course. The geometry of the fairway can also be useful for other purposes. The width will for instance indicate the size of deviation in position that is reasonable. The width of the fairway therefore contains useful information that will be used for association purposes.

\subsection{Model for movement and uncertainty}

The idea behind the model is to capture the main movements and the uncertainty in the time of departure and arrival, or speed. The objective is not a stochastic model that inherits all possible dynamic and statistic complexity.

We assume that in the mean route coordinate system, the instantancous speed is a mean reverting process. The model is formulated in a Kalman filter framework so measure updating is easy to handle each time a report about position is received.

The system model is as follows ${ }^{1}$ :

$$
x_{k}=\left(\begin{array}{ccc}
1 & 0 & 0 \\
\frac{\Delta t_{k}}{T} & 1-\frac{\Delta t_{k}}{T} & 0 \\
0 & \Delta t_{k} & 1
\end{array}\right) x_{k-1}+\left(\begin{array}{c}
0 \\
w_{k-1} \\
0
\end{array}\right)
$$

where $x_{k}=\left(\begin{array}{lll}m_{k} & v_{k} & d_{k}\end{array}\right)^{t}$ and $m_{k}$ represents mean velocity, $v_{k}$ instantaneous velocity and $d_{k}$ represents distance travelled at time $k$ in the mean route coordinate system. $T$ is a time constant. We assume that $x$ is normally distributed, and $\left\{w_{k}\right\}$ being a sequence of independent normally distributed variables. The process noise, represented by $w$, models the uncertainty with respect to projection of positions on the route and the length of the route. The observed process $z$ is given by

$$
z_{k}=H_{k} \cdot x_{k}
$$

where $H_{k}$ is a three dimensional matrix with the following properties:

- If there is no observation at time $k$ then $H_{k}$ is the zero matrix.

- If only distance is observed at time $k$ the element $\left\{H_{k}\right\}_{3,3}$ is equal to 1 , the other elements are zero.

- If both distance and instantaneous velocity is observed, $\left\{H_{k}\right\}_{2,2}$ and $\left\{H_{k}\right\}_{3,3}$ is equal to 1 and the remaining elements zero.

We assume that the vessels report their position under certain conditions. When a report is received, the position is projected on the mean route using the method described in section 4.1. The distance corresponding to this position is then used as an observation to update the estimate. The Kalman filter equations in discrete time that give the optimal estimate can be found for instance in Gelb (1974).

In addition to departure and destination location we also assume that we have:

- Some prior estimate about the time of departure, $t_{\text {dep }}$.

- Some prior estimate about the mean velocity, $m$.

'Thanks to Mr. Frank Mulder, Thales Nederland B.V., for making us aware of an error in the system equations in the original paper. 
The uncertainty of the time of departure is modelled as uncertainty in the initial position (distance) $d_{0}$, which can be approximated by the equation:

$$
d_{0} \approx m \cdot t_{\text {dep }}
$$

It is assumed that the mean velocity and the time of departure are independent. Hence, $E\left[m \cdot t_{d e p}\right]=\bar{m} \cdot \bar{t}_{d e p}$ and

$$
\operatorname{var}\left(d_{0}\right)=\operatorname{var}(m) \cdot \operatorname{var}\left(t_{d e p}\right)+\operatorname{var}(m) \cdot \bar{t}_{d e p}^{2}+\operatorname{var}\left(t_{d e p}\right) \cdot \bar{m}^{2}
$$

where $\bar{t}_{d}$ is the expected time of departure and $\bar{m}$ is the expected mean velocity. We assume that $d_{0}$ is normally distributed. The interpretation of a negative distance is that the vessel has not departed.

\section{The multiple hypothesis association formulation}

As mentioned previously the typical number of unknown vessels in an area is much larger than the number of vessels with a known route plan. This can lead to an underdetermined association problem, i.e. there is more than one possible solution to the problem.

State of the art radar tracking in e.g. air defence systems uses multiple hypothesis tracking to solve a difficult association problem due to highly manoeuvring targets and radar detections with a low signal-to-noise ratio (Blackman et al., 1996). We have taken a similar approach and look at information collected over a large time frame and postpone the association decisions until a solution has gained credibility.

To simplify the association problem we have made the following assumptions:

- A vessel within radar coverage is always detected

- The tracks are stable (constant track quality)

- We have no false tracks

- There are no redundant tracks (the track-to-track association is perfect)

We also assume that the reports on a vessel cause no association problems, which reduces the problem to make hypotheses about the origin of unidentified radar tracks. The origin of an unidentified track is then either:

1. A known vessel with a route plan

2. An unknown vessel

\subsection{Association metrics}

A measure of the likelihood of the association between vessel $i$ with a route plan and a track $j$ may be formulated as:

$$
p_{i j}=\frac{e^{-\frac{d_{i j}^{2}}{2}}}{(2 \pi)^{M / 2} \sqrt{\left|S_{i j}\right|}}
$$

where $S_{i j}$ is the covariance matrix $S_{i j}=S_{i}+S_{j}$ and $M$ is $\operatorname{dim}(z) . d_{i j}$ is the euclidian distance measure

$$
d_{i j}^{2}=(z-H \bar{x})^{T} S_{i j}^{-1}(z-H \bar{x})
$$


which e.g. can be found in Hall (1992). The threshold for deciding that a track is an unknown vessel can be formulated as

$$
p=1-\frac{\beta_{K V}}{\beta_{T V}}
$$

where $\beta_{K V}$ is the average density of known vessels in an area and $\beta_{T V}$ is the average density of vessels in an area. Equation (7) could be used as a threshold to decide when not to associate the track and leave it unknown.

We have chosen not to associate probabilities with the width of the fairway, but only use it as a constraint on the movement of the vessel.

\subsection{Hypothesis generation}

The approach chosen is generating hypotheses that consist of sets of consistent associations, which are ranked by a score function. A hypothesis is a possible solution of the total association problem between unknown tracks and known vessels. Hypothesis management is a complex problem, and implementing it is a time consuming task. This motivates for an approach that facilitates a high level description of the association problem. One such technique is constraint programming (Tsang, 1993). To be able to use this technique we have to formulate the problem as a Constraint Satisfaction Problem (CSP).

A CSP consists of variables where each variable has a domain of possible values. A set of constraints restricts the values that the variables can simultaneously take. We have chosen to represent the unidentified tracks as variables and the known vessels as domain values.

A constraint has to be fulfilled before the association between a track and a known vessel can take place. An objective function is used in order to find the best possible solution.

The constraints that must be obeyed are:

- The vessel is inside the fairway.

- The course is within possible range given by the fairway, and the vessel is sailing in the direction of the fairway.

- The speed satisfies the constraint given by the vessel speed range.

- Only one track can be associated with a vessel at one point in time.

The objective function that is to be optimized contains the following elements:

- The position of a track related to the estimated position of the known vessel.

- The speed of the track compared with the average vessel speed.

- The course of the track related to movement along the fairway.

The two last items has to be treated specially and are not combined in equation (6). The reason for this is that the estimated vessel speed is the average speed along the mean route, and this may not correspond to the actual speed of the vessel. The use of course information is neither straight forward due to the fact that vessels will try to move along the shortest path.

Even though a vessel may only be associated with one track at a given point in time, it may be associated with a set of tracks that do not overlap in time. Thus a hypothesis consists of associations of a vessel with both current and lost tracks. It is necessary to include previous tracks in order to use the track to update the estimated movement of the vessel according to the route model. 
In contrast to multiple hypothesis tracking we in this application have asynchronous data, and we are therefore not able to calculate the score values of all the hypotheses or the different associations in a hypothesis at a common point in time.

An advantage of using constraint programming is that it is easy to extend the model to utilize additional information without altering the algorithm. One possible extension is to allow a vessel to have alternative routes in case there is doubt about which route that is to be followed. This can be implemented by adding the route as a variable attached to the vessel.

The method presented has been integrated in a tool that simulates the surface coverage of various sensor platforms in a user-defined scenario (Mevassvik, 1996). This tool has also produced the data that has been input to the algorithm. ILOG Solver (ILOG, 1998) has been used for the constraint programming part of the simulation.

\section{An example using information from merchant shipping}

This section contains an example scenario that has been simulated. The example is extreme in the sense that the unknown vessels follow almost exactly the same route as the known vessels.

The current implementation of the presented method does only use initial and current position of the track when searching for a solution. Due to this a track can temporarily be outside the fairway but yet be part of a solution some time later. The position of the tracks is not used when updating the estimated movement, which could have produced better results.

The simulated scenario consists of two known merchant vessels, in addition to some unknown traffic. The known merchant vessels have to forward certain information prior to calling ports. We get an arrival message containing a route plan that tells which harbours they are going to visit and a time plan. From this we have made estimated routes (see Figure 2).

The following messages are sent from the vessels during the simulations:

- A notification message upon entering/leaving the territorial waters.

- A notification message when passing by one of six reporting points along the Norwegian coast.

In addition to this the local Harbour Authorities registers when the vessels are calling at the port and upon leaving.

There are two coastal radars in the area that has an approximate coverage shown as circles in Figure 2.

The tracks from these radars are combined to form unique global tracks by a simulated fusion centre.

\subsection{Simulation results}

The simulation of a merchant vessel is shown in Figures 3 and 4. Figure 3 shows the tracks of three unknown vessels that move along with the vessel we are looking for. The circles in the figure indicate that we have a correct association. The unknown vessels have slightly different speed and have started at different times than the known vessel, but follow the same route. We see that most of the time the track of the known vessel and the tracks of the unknown vessels are sufficiently separated. 


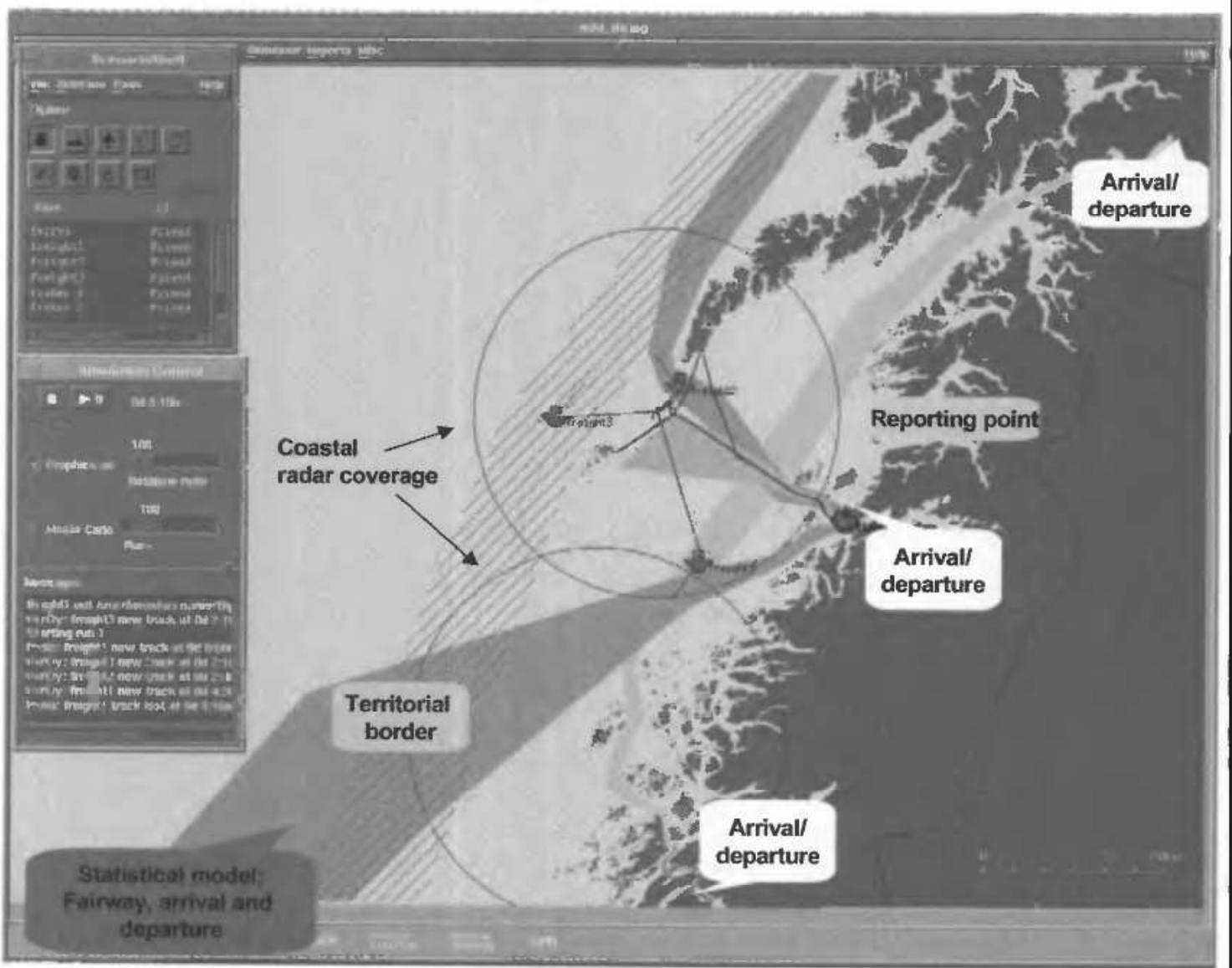

Figure 2. The scenario used in the simulations.

Figure 4 shows the progress of the quality of the various associations between tracks and the estimated voyage of the known vessel. The track of the vessel we are looking for is drawn using a bold line.

Figure 5 shows the uncertainty in the estimated position of the vessel given by the route model. The gate which shall contain the vessel span the entire width of the fairway. For the estimated distance a $2 \sigma$ confidence interval is shown.

Figure 6 shows a vessel sailing between two ports, reporting its position upon leaving the port and when passing by the reporting point indicated in Figure 2. There are four more unknown vessels following the same route. From Figure 6 we can see that during a large interval before the position report is received, a false association is made. The reason for this can be seen in Figure 7, which shows the development of the association metric for all tracks as a function of time (bold line represents correct track). When the vessel leaves the port, the Harbour Authorities send a message, which leads to a high score in the beginning. As the uncertainty increases the measure becomes less confident until the vessel sends a position report after approximately 6.5 hours.

Between 3 to 6.5 hours upon departure there are other vessels catching up with the known vessel and vice versa. The tracks of these vessels are at certain times more credible than the correct track until the position report is received. 


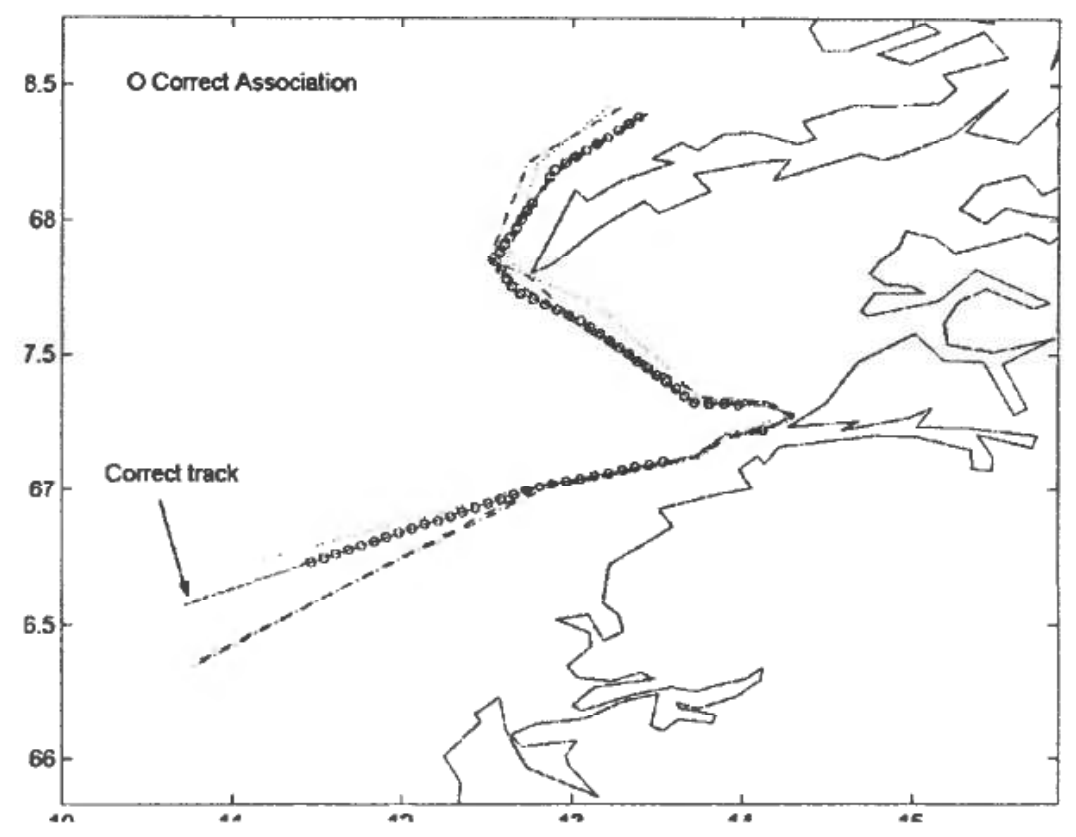

Figure 3. Four vessels following the same route.

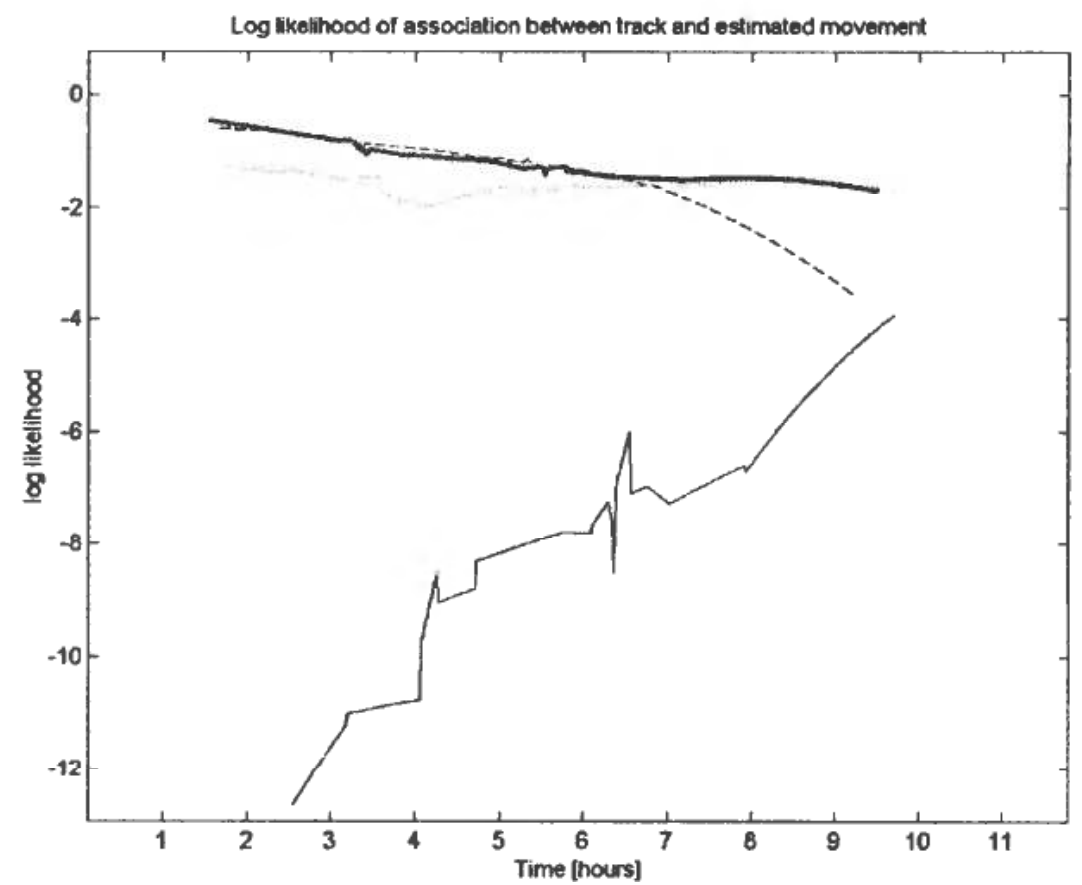

Figure 4. The progress of the quality of the associations between the tracks and estimated movement. 


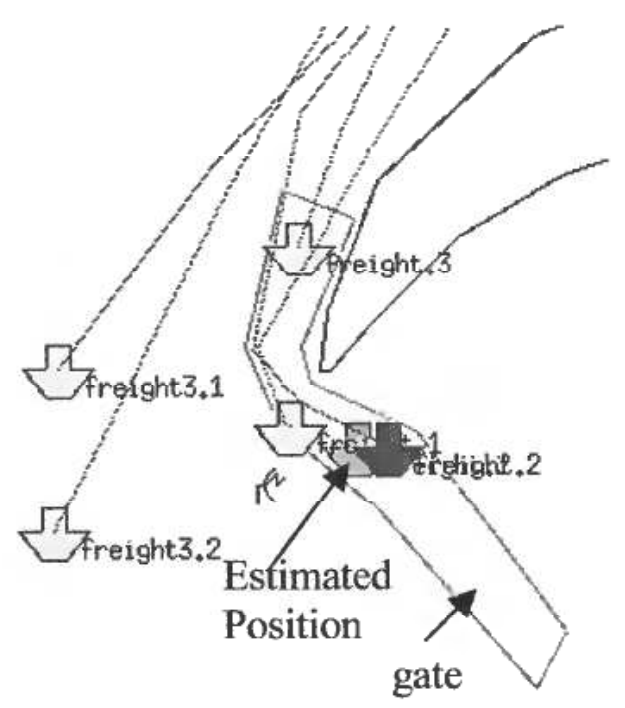

Figure 5. The position estimate given by the route model.

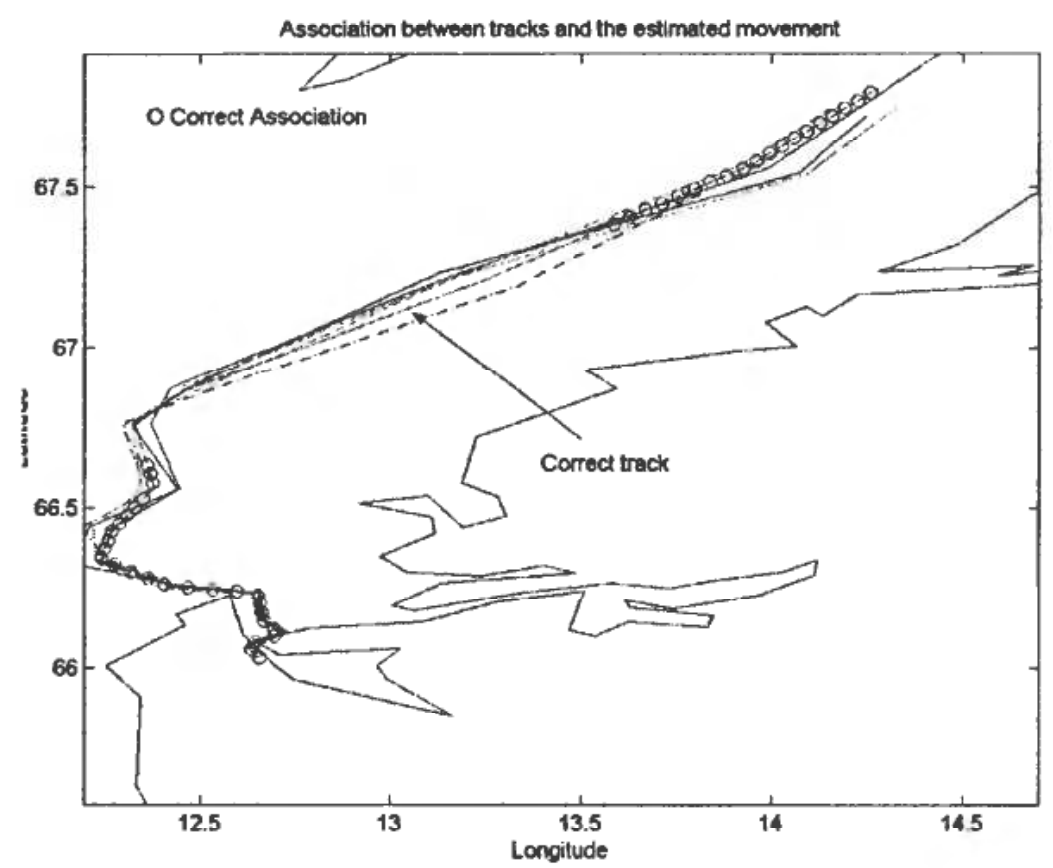

Figure 6. A merchant vessel sailing between two ports.

Figure 7 also illustrates the potential complexity in finding the correct track when there are a number of vessels in the area that follow approximately the same route as the known vessel.

\section{Conclusions}

This paper has presented a method for utilising a priori information on the movement of a vessel. 


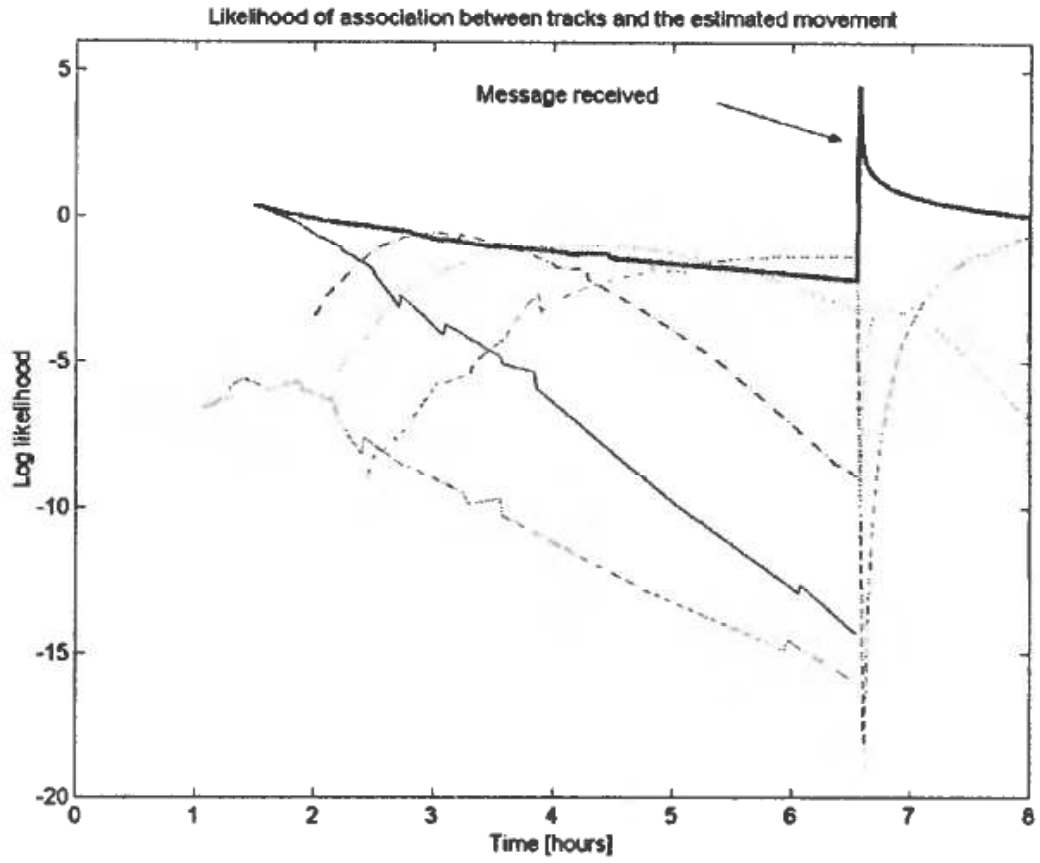

Figure 7. The association metric as a function of time.

The method consists of using a statistical model of the movement of a vessel. This model is updated with position reports from the vessel and then associated with radar tracks using a multiple hypothesis approach. Constraint programming has been used to manage hypotheses and finding the best solution.

There are several applications for the approach that is proposed:

- As a control mean to monitor if vessels are sailing according to a given route plan (consistency checking).

- As an aid to help to do classification of radar tracks, and to increase the completeness of a surface picture.

- A more sophisticated model for displaying planned movements (PIM-tracks) for vessels by displaying the uncertainty in the track.

\section{References}

GelB, A. (editor) (1974), Applied Optimal Estimation, M.I.T. Press.

Blackman, S. S., Dempster, R. J. \& Sylvain, B. (1996), Application of Multiple Hypothesis Tracking to Multi-radar Air defence systems, AGARDograph 337, Multi-Sensor MultiTarget Data fusion, pp. 96-120.

Hall, D. L. (1992), Mathematical Techniques in Multisensor Data Fusion, Artec House.

Tsang, E. (1993), Foundations of Constraint Satisfaction, Academic Press.

MEVASSVIK, O. M. (1996), SensorSim - a simulation program for maritime surveillance, Forsvarets forskningsinstitutt (in Norwegian).

ILOG (1998), ILOG Solver 4.3 User's Manual, ILOG. 